\title{
A case of late dissection of aorta post aortic valve replacement
}

\begin{abstract}
Dissection of aorta post aortic valve replacement (AVR) is a potentially fatal and rare complication. Presence of bicuspid valve, aortic regurgitation, systemic hypertension and marfans syndrome prior to surgery are predictors for such events post aortic valve replacement. The measurement of aortic root and ascending aorta diameter by transesophageal echocardiography (TEE), computed tomography (CT) or magnetic resonance imaging (MRI) helps to choose which patients should undergo prophylactic ascending aorta graft along with AVR to prevent such catastrophes in future.
\end{abstract}

Keywords: aortic valve replacement, transesophageal echocardiography, computed tomography, AVR, MRI
Volume I4 Issue 2 - 202 I

\section{Ankush Sachdeva}

Department cardiology, Fortis Escorts Heart Institute, Indian

Correspondence: Ankush Sachdeva, Fortis Escorts Heart Institute, Okhla, New Delhi, India, Tel 99| I67|254,

Email ankushsachdeva0@gmail.com

Received: April 03, 2021 | Published: April 30, 2021
Abbreviations: AVR, aortic valve replacement; TEE, transesophageal echocardiography; CT, computed tomography; MRI, magnetic resonance imaging

\section{Introduction}

Type A dissection is seen in $0.6 \%$ of patients late after aortic valve replacement and $13 \%$ of patients with type A dissection have a history of AVR. ${ }^{1}$ Patients with an aortic root diameter of $\geq 43 \mathrm{~mm}$ should be assessed for potential risk factors for late dissection and are likely to benefit from a prophylactic aortic surgery. ${ }^{2}$

\section{Case report}

A 43year old male had undergone an aortic valve replacement using bileaflet mechanical prosthesis for bicuspid aortic valve causing severe aortic stenosis and a moderate aortic regurgitation at an outside hospital five years ago. He presented to our emergency department with history of acute onset of sudden severe chest pain radiating to interscapular region and back with an episode of vomiting and transient loss of consciousness leading to a fall one hour ago.

On examination, he was conscious oriented, responding to verbal commands and moving all limbs. His pulse rate was 105/minute, blood pressure $-100 / 70 \mathrm{mmHg}$. Peripheral pulses were feeble. ECG revealed a sinus tachycardia. A prosthetic valve click was heard on cardiac auscultation. There were normal vesicular breath sounds heard bilaterally .No adventitious sounds or murmur was heard. Chest $\mathrm{x}$-ray was suggestive of mediastinal widening.

A transthoracic echocardiogram was done which revealed a normal functioning mechanical prosthesis at aortic position with no valvular or paravalvular regurgitation. A huge aneurysm of ascending aorta with a maximum diameter of $10 \mathrm{~cm}$ was noted (Figure 1A). There was a strong suspicion of a dissection flap along the aneurysm. A small loculated pericardial collection was seen anteriorly. An urgent CT aortogram (Figure 1B) was done which confirmed a large ascending aorta aneurysm with a dissection flap arising near to the origin of right coronary artery and extending into aortic arch. There was no dissection of the coronaries. There was a focal rupture of distal ascending aorta with a pericardial leak suggesting a contained rupture. Minimal ascites and minimal pleural effusion was noted. Patient was taken up for an urgent ascending aorta repair and graft but unfortunately he expired during surgery.

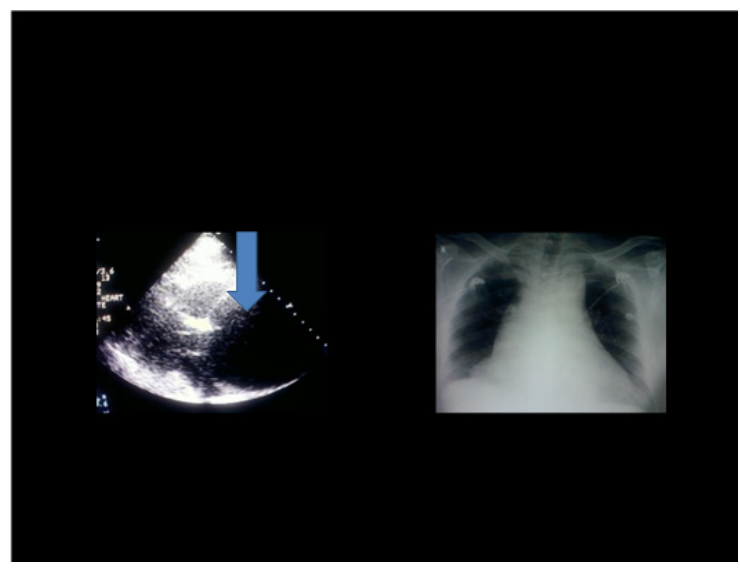

Figure I A Parasternal long axis view (PLAX) on transthoracic echocardiogram showing ascending aorta aneurysm (arrow mark) and chest $\mathrm{x}$-ray showing mediastium widening (right).

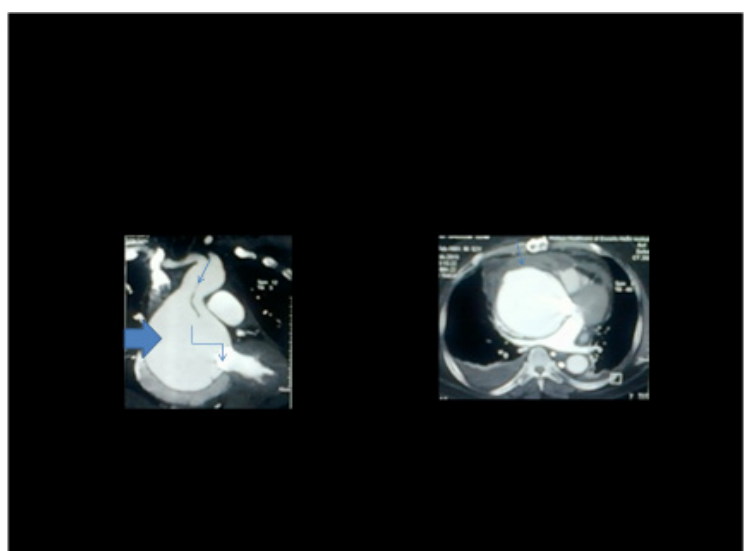

Figure IB CT scan showing large ascending aorta aneurysm (bold arrow), dissection flap (down arrow), aortic mechanical prosthesis (bend square arrow)on left image and pericardial collection (arrow mark) suggesting a contained rupture on right image.

\section{Discussion}

Aortic regurgitation in presence of moderate aortic root dilatation reflects an aortic root disease and isolated AVR is an incomplete 
treatment. In general aortic diameter of $<50 \mathrm{~mm}$ carries a low risk of dissection or rupture. ${ }^{3}$ Aortic wall fragility in combination with aortic thinning or aortic regurgitation carries a $64 \%$ or $79 \%$ probability of dissection which increases to $96 \%$ in presence of all these factors. There has been a high prevalence of aortic regurgitation (92\%), arterial hypertension (57\%), cystic medial necrosis (39\%) and bicuspid aortic valves (24\%) in these cases while paravalvular leak after AVR plays a major role in development of non-dissecting aneurysms. ${ }^{4}$

\section{Acknowledgments}

None.

\section{Conflicts of interest}

Author has no pertinent financial interests with the subject matter or materials discussed.

\section{Funding}

None.

\section{References}

1. Muna WF, Spray TL, Morrow AG, et al. Aortic dissection after aortic valve replacement in patients with valvular aortic stenosis. $J$ Thorac Cardiovasc Surg.1977;74(1):65-69.

2. Pieters FAA, Widdershoven JW, Gerady AC, et al. Risk of aortic dissection agter aortic valve replacement. Am J Cardiol. 1993;72(14):1043-1047.

3. Platis IE, Qader MA, Coady MA, et al. Should a moderately dilated aorta be resected at the time of routine AVR ? Circulation. 1998;98(suppl 1):1-266.

4. Derkac W, Laks H, Cohn LH, et al. Dissecting aneurysm after aortic valve replacement. Arch Surg.1974;109(3):388-390. 